\title{
Co-crystallization of hepatitis C virus NS3/4A inhibitors and SARS-CoV-2 main protease using high density acoustic droplet ejection (ADE)
}

\author{
Babak Andi ${ }^{1,3}$, Dale F. Kreitler ${ }^{1}$, Desigan Kumaran ${ }^{2,3}$, Alexei S. Soares ${ }^{1}$, Jantana Keereetaweep ${ }^{2}$, Jean Jakoncic ${ }^{1}$, \\ Wuxian Shi ${ }^{1}$, Martin R. Fuchs ${ }^{1}$, John Shanklin ${ }^{2}$, Sean McSweeney ${ }^{1,2,3}$ \\ ${ }^{1}$ Center for BioMolecular Structure, NSLS-II, Brookhaven National Laboratory, Upton, NY 11973-5000, United States, \\ ${ }^{2}$ Biology Department, Brookhaven National Laboratory, Upton, NY 11973-5000, United States, \\ ${ }^{3}$ National Virtual Biotechnology Laboratory (NVBL), US Department of Energy, Washington, DC, United States \\ bandi@bnl.gov
}

COVID-19 pandemic is a great threat to the general and global public health and economy. The rapid development of new antiviral compounds and vaccines is needed to control the current pandemic as well as to prepare for the emergence of new variants. Among the proteins encoded by the SARS-CoV-2 genome, $\mathrm{M}^{\text {pro }}$ is one of the primary drug targets due to its essential role in maturation of the viral polyprotein. In this study, we describe a high-density acoustic droplet ejection (ADE) method for co-crystallization of $\mathrm{M}^{\mathrm{pro}}$ ligand complexes using only $40 \mathrm{~nL} \mathrm{M}{ }^{\text {pro }}$ solution. Also, we will briefly describe crystallographic data from crystals obtained using $\mathrm{ADE}$ and other methods as evidence that three clinically approved anti hepatitis $\mathrm{C}$ virus (HCV) drugs are capable of covalent binding to the $\mathrm{M}^{\text {pro }}$ Cys145 catalytic residue in the active site (Fig. 1). Activities of the National Virtual Biotechnology Laboratory (NVBL) for the design and development of new antiviral inhibitors for SARS-CoV-2 is briefly discussed.



Figure 1. Crystal structure of SARS-CoV-2 main protease in complex with covalent inhibitor Telaprevir at 1.48 $\AA$ (PDB: 7K6D)

Keywords: SARS-CoV-2; main protease; acoustic droplet ejection; Telaprevir

This research was supported by the DOE Office of Science through the National Virtual Biotechnology Laboratory (NVBL) with funding from the Coronavirus CARES Act and additional funding was provided by Brookhaven National Laboratory (BNL) for research on COVID-19 (LDRD 20-042). This research used AMX and FMX beamlines of the National Synchrotron Light Source II, a U.S. Department of Energy (DOE) Office of Science User Facility operated for the DOE Office of Science by Brookhaven National Laboratory under Contract No. DE-SC0012704. The Center for BioMolecular Structure (CBMS) is primarily supported by the National Institutes of Health, National Institute of General Medical Sciences (NIGMS) through a Center Core P30 Grant (P30GM133893), and by the DOE Office of Biological and Environmental Research (KP1605010). 